\title{
Regulatory T cells induced by B cells: a novel subpopulation of regulatory $T$ cells
}

\author{
Chien-Hui Chien ${ }^{1}$ and Bor-Luen Chiang ${ }^{1,2^{*}}$
}

\begin{abstract}
Regulatory $T$ cells play a crucial role in the homeostasis of the immune response. In addition to $C D 4^{+} F o x p 3^{+}$ regulatory $T$ cells, several subsets of Foxp3 ${ }^{-}$regulatory $T$ cells, such as $T$ helper 3 (Th3) cells and type 1 regulatory $T$ (Tr1) cells, have been described in mice and human. Accumulating evidence shows that naive B cells contribute to tolerance and are able to promote regulatory $T$ cell differentiation. Naïve B cells can convert $C D 4^{+} C D 25^{-} T$ cells into CD25 ${ }^{+}$Foxp3 $^{-}$regulatory $T$ cells, named Treg-of-B cells by our group. Treg-of-B cells express LAG3, ICOS, GITR, OX40, PD1, and CTLA4 and secrete IL-10. Intriguingly, B-T cell-cell contact but not IL-10 is essential for Treg-of-B cells induction. Moreover, Treg-of-B cells possess both IL-10-dependent and IL-10-independent inhibitory functions. Tregof-B cells exert suppressive activities in antigen-specific and non-antigen-specific manners in vitro and in vivo. Here, we review the phenotype and function of Foxp3 ${ }^{+}$regulatory T cells, Th3 cells, Tr1 cells, and Treg-of-B cells.
\end{abstract}

Keywords: Regulatory T cells, Lymphocyte-activation gene 3, Programmed cell death protein 1, Inducible T-cell co-stimulator, Interleukin 10, Cytotoxic T lymphocyte-associated antigen-4, Treg-of-B cells

\section{Background}

Regulatory $\mathrm{T}$ cells are a therapeutic strategy for immune dysregulated diseases and a potential target for cancer immunotherapy. In addition to $\mathrm{CD}^{+}{ }^{+} \mathrm{Foxp}^{+}$regulatory $\mathrm{T}$ (Treg) cells, studies have emphasized the roles of CD4 ${ }^{+}$Foxp $3^{-}$regulatory $\mathrm{T}$ cells, such as TGF- $\beta$-producing $\mathrm{T}$ helper 3 (Th3) cells, IL-10-producing type 1 regulatory $\mathrm{T}(\mathrm{Tr} 1)$ cells, and others. Accumulating evidence demonstrate that naïve $\mathrm{B}$ cells possess the ability to promote naïve $\mathrm{CD} 4^{+} \mathrm{T}$ cells into $\mathrm{CD}^{2} 5^{+}$Foxp3 ${ }^{-}$regulatory $\mathrm{T}$ cells with the expression of lymphocyte activation gene-3 (LAG3, CD223), inducible co-stimulator (ICOS, CD278), programmed cell death protein 1 (PD1, CD279), and glucocorticoid-induced TNFR family-related protein (GITR). B-cell-induced $\mathrm{CD}^{+}{ }^{+}$Foxp3 $3^{-}$regulatory $\mathrm{T}$ cells exert the inhibition through both IL-10-independent and cell-cell contact-dependent mechanisms, although they also show IL-10-mediated suppression. Furthermore, these B cell-induced regulatory $\mathrm{T}$ cells protect mice from several immune disorders, including graft-versus-host disease, experimental allergic asthma, collagen-induced

\footnotetext{
* Correspondence: gicmbor@ntu.edu.tw

${ }^{1}$ Graduate Institute of Clinical Medicine, National Taiwan University, Taipei City 10048, Taiwan, Republic of China

${ }^{2}$ Department of Medical Research, National Taiwan University Hospital, Taipei City 10002, Taiwan, Republic of China
}

arthritis, and inflammatory bowel disease. Here, we review the phenotypes and functional mechanisms of thymus-derived and peripherally derived $\mathrm{CD} 4^{+} \mathrm{Foxp} 3^{+}$ regulatory $\mathrm{T}$ cells, Th3 cells, $\operatorname{Tr} 1$ cells, B-cell-induced Foxp3- regulatory $\mathrm{T}$ cells, and B-cell-induced Foxp $3^{+}$ regulatory $\mathrm{T}$ cells. The present article focuses on B-cellinduced $\mathrm{CD} 4^{+}$Foxp $3^{-}$regulatory $\mathrm{T}$ cells, which we have named Treg-of-B cells.

\section{Main text \\ $\mathrm{CD}^{+}{ }^{+}$Oxp $^{+}{ }^{+}$regulatory $\mathrm{T}$ cells}

Sakaguchi et al. demonstrated that $\mathrm{CD} 4^{+} \mathrm{CD} 25^{+} \mathrm{T}$ cells contributed to maintaining self-tolerance in a nonantigen-specific manner [1]. Immune dysregulation, polyendocrinophathy, enteropathy X-linked (IPEX) syndrome is a recessive immune disorder. Reports showed that IPEX is caused by mutations of FOXP3 gene, which is orthologouse of the Foxp3 gene mutated in scurfy mouse [2-4]. Further studies demonstrated that Foxp3 expressed predominantly in $\mathrm{CD} 4{ }^{+} \mathrm{CD} 25^{+} \mathrm{T}$ cells than $\mathrm{CD} 4^{+} \mathrm{CD} 25^{-} \mathrm{T}$ and $\mathrm{CD} 19^{+} \mathrm{B}$ cells. Moreover, retroviral transduction of Foxp3 in naïve $\mathrm{CD} 4^{+} \mathrm{CD} 25^{-} \mathrm{T}$ cells converted these cells toward Treg cells phenotype. Thus, Foxp3 has been identified as the master transcription factor of Treg cells [5]. 


\section{Thymus-derived Foxp $3^{+}$regulatory T cells}

In addition to Foxp3, thymus-derived $\mathrm{CD} 4{ }^{+} \mathrm{CD} 25^{+}$Foxp3

+ regulatory $\mathrm{T}$ (tTreg) cells highly expressed Helios, cytotoxic $\mathrm{T}$ lymphocyte-associated antigen-4 (CTLA4, CD152), neuropilin-1, GITR, galectin-1, IL-10, and granzyme B [6]. tTreg cells could be activated in an antigenspecific fashion and exerted suppressive activity in a non-antigen-specific fashion [7]. tTreg cells produced many inhibitory cytokines, including TGF- $\beta 1$, IL-10, and IL-35, to downregulate immune responses [8]. Furthermore, tTreg cells exhibited cell-cell contact-dependent suppression via latency-associated peptide (LAP) [9], CD39 (ectonucleoside triphosphate diphosphohydrolase1, ENTPD1) and CD73 (ecto-5' -nucleotidase) [10], and cytosolic cyclic adenosine monophosphate (cAMP) [11]. Reports showed that $\mathrm{t}$ Treg cells induced effector $\mathrm{T}$ cell apoptosis via various pathways, including deprivation of IL-2 and IL-7 [12], disruption of effector cell membrane integrity by granzyme B [13], galectin-1-induced apoptosis [14], and the engagement of TNF-related apoptosis inducing ligand (TRAIL)-death receptor 5 (DR5) [15]. Additionally, tTreg cells inhibited effector $\mathrm{T}$ cell activation via downregulation of costimulatory molecules on DCs through CTLA4 [16] and LAG3 [17]. These studies indicate that tTreg cells are a polyclonal population, and the above mentioned complicated mechanisms result in maximal immunosuppression during homeostasis.

\section{Peripherally derived Foxp $3^{+}$regulatory $\mathrm{T}$ cells}

Foxp $^{+}$regulatory $\mathrm{T}$ cells induced in vivo are called peripherally derived regulatory $\mathrm{T}$ (pTreg) cells and those generated in vitro are called in vitro-induced regulatory $\mathrm{T}$ (iTreg) cells [18]. Studies demonstrated that CD4 ${ }^{+}$Foxp3 ${ }^{-} \mathrm{T}$ cells differentiated into Foxp $3^{+} \mathrm{CD} 25$ ${ }^{+} \mathrm{CD} 45 \mathrm{RB}^{\text {low }}$ anergic $\mathrm{T}$ cells with suppressive functions in the presence of TGF- $\beta 1$ in vitro as well as in vivo [19] and rescue Foxp3-deficient scurfy mice [20]. In the absence of tTreg cells, oral antigen administration induced the generation of $\mathrm{CD} 4^{+} \mathrm{CD} 25^{+} \mathrm{Foxp} 3^{+}$regulatory $\mathrm{T}$ cells in a TGF- $\beta 1$-dependent manner [21]. Gut-associated lymphoid tissue $\mathrm{CD}_{103^{+}}$DCs played an important role in the de novo conversion of naïve $\mathrm{T}$ cells into pTreg cells, and retinoic acid facilitates that process [22]. Additionally, lung-resident tissue macrophages expressed retinal dehydrogenases, and TGF- $\beta 1$ promoted pTreg cell induction under steady-state conditions [23]. Evidence has shown that the tumor environment induced pTreg cell generation to escape immune clearance [24]. One report demonstrated that tTreg and pTreg cells shared similar phenotypes, and neuropilin-1 serving as a surface marker to distinguish tTreg cells from pTreg cells [25].

\section{$\mathrm{CD}^{+}{ }^{+}$Foxp3 $3^{-}$regulatory $\mathrm{T}$ cells}

The most well-defined Foxp3 ${ }^{-}$regulatory $\mathrm{T}$ cells are Th3 cells and $\operatorname{Tr} 1$ cells. Th3 cells have been identified as TGF- $\beta$-producing $C D 4^{+} \mathrm{LAP}^{+} \mathrm{T}$ cells exhibiting TGF- $\beta$ mediated suppression [26]. Tr1 cells have been characterized by the higher production of IL-10 and IL10-mediated suppressive functions [27].

\section{T helper 3 cellsl}

Th3 cells were first found in mesenteric lymph node $\mathrm{CD}^{+}{ }^{+} \mathrm{T}$ cells as single cell clones producing TGF- $\beta 1$ after oral administration of self-antigen [28]. Oida et al. found that primary purified $\mathrm{CD} 4^{+} \mathrm{CD} 25^{-} \mathrm{LAP}{ }^{+}$regulatory $\mathrm{T}$ cells protected mice from T-cell-induced colitis in a TGF- $\beta 1$-dependent manner [29]. Tumor environment $\mathrm{CD} 4^{+} \mathrm{CD} 25^{-} \mathrm{CD} 9^{+} \mathrm{Foxp}^{-} \mathrm{LAP}^{+} \mathrm{T}$ cells expressed IL-2 receptor $\beta$ chain, produced TGF- $\beta 1$, and exerted TGF$\beta 1$-mediated functional activity [30]. Gandhi et al. showed that human peripheral $\mathrm{CD} 4^{+} \mathrm{LAP}^{+} \mathrm{Foxp}^{-} \mathrm{CD} 69^{+}$ $\mathrm{T}$ cells exhibited TGF- $\beta 1$ - and IL-10-dependent suppression in the periphery in healthy individuals [31]. Furthermore, human $\mathrm{CD}^{+}{ }^{+} \mathrm{CD} 25^{+} \mathrm{LAP}^{+}$Foxp3 ${ }^{-} \mathrm{T}$ cells in colorectal tumors expressed LAG3 and exhibited inhibitory functions through TGF- $\beta 1$ and IL-10 [32]. To date, the specific transcription factor for Th3 cells remains to be identified.

\section{Type 1 regulatory T cells}

The first study on $\operatorname{Tr} 1$ cells reported that naïve $\mathrm{T}$ cells repeated stimulation with peptide-pulsed splenocytes in the presence of IL-10 induced IL-10-producing $\mathrm{CD} 4^{+} \mathrm{T}$ cells with suppressive ability and hypoproliferative ability [33]. Akbari et al. demonstrated that bronchial DCs promoted Tr1 cells in vitro in an IL-10-and ICOS/ ICOS ligand (ICOSL)-dependent manner in the context of nasal tolerance [34]. By microarray analysis $\operatorname{Tr} 1$ and Th0 cell clones, CD49b, LAG3, and CD226 have been identified as the surface markers of $\operatorname{Tr} 1$ cells [35].

It has been shown that c-Maf transactivated IL-10 expression under $\mathrm{CD} 4^{+}$Th17 polarization conditions [36]. Aryl hydrocarbon receptor (AhR) and c-Maf facilitated IL-10 production in $\mathrm{CD}^{+} \mathrm{T}$ cells in an IL-27dependent fashion $[37,38]$. Another study reported that c-Maf, IL-21, and ICOS were essential for IL-27-induced $\operatorname{Tr} 1$ cell generation [39]. Consistent with these observations, Awasthi et al. showed that $\mathrm{CD} 4^{+} \mathrm{Foxp}^{+}$regulatory T cell-educated DCs produced IL-27 and promoted Tr1 cell generation [38]. Nasal anti-CD3 $\varepsilon$ antibody treatment induced the expression of IL-10, IL-27, and TGF- $\beta$ in nasal tolerogenic DCs, which further facilitated $\operatorname{Tr} 1$ cell generation through c-Maf, IL-21, and AhR [40]. Orally antigen treated tolerogenic Peyer's patch DCs increased the production of IL-10 and IL-27 and promoted the induction of $\operatorname{Tr} 1$ cells [41]. Carrier et al. reported that 
constitutive ectopic expression of GITR ligand (GITRL) on $\mathrm{MHCII}^{+}$APCs increased IL-27 production and further upregulated the expression of c-Maf and IL-10 in T cells [42].

In addition to cytokines, reports have demonstrated that $\operatorname{Tr} 1$ cells could be induced by different proteins, different APCs, and different types of T cells. Galectin-1 promoted IL-10 expression in CD $4^{+} \mathrm{T}$ cells in an APCindependent pathway by binding to CD45 on T cells and inducing the expression of c-Maf and AhR [43]. In vitro activation of $\mathrm{CD} 4^{+} \mathrm{CD} 44^{\text {hi }}{ }^{-}$oxp $3^{-} \mathrm{T}$ cells through antiCD3/CD28 antibodies and IL-2 generated CD49b-, LAG3-, c-Maf-, and AhR-expressing Tr1 cells [44]. Nie et al. found that long-term stimulation of lipopolysaccharide (LPS) conferred ICOSL expression in bone marrow-derived mast cells through NF- $\mathrm{kB}$, subsequently promoting $\operatorname{Tr} 1$ cell development [45]. These reports suggest that the generation mechanisms for $\operatorname{Tr} 1$ cells consist of a fine-tuning program.

\section{$B$ cells in tolerance induction}

$B$ cells have been shown to have a role in the fine equilibrium for immune tolerance. Genetically B-celldeficient mice delayed recovery from experimental autoimmune encephalomyelitis and suggested B cells might contribute to immune modulation [46]. Collagen fragments expressed on B cell MHC class II sufficiently delayed the onset and decreased the severity of arthritis [47]. The role of B cells in oral tolerance has been investigated because B-cell-deficient mice exhibit a defective oral tolerogenic response characterized by lower levels of IL-10 and TGF- $\beta$ in the spleen and gut-associated lymphoid tissues [48]. Gutgemann et al. showed that B cells interacted with $\mathrm{T}$ cells at the B-T border in the spleen after $4 \mathrm{~h}$ of oral administration of proteins [49]. Furthermore, orally antigen treated B cells have an enhanced ability to induce $\mathrm{CD} 4^{+}$regulatory $\mathrm{T}$ cells in vitro [50]. Anterior chamber-associated immune deviation was characterized by antigen-specific downregulation of the immune response to antigen occurs in the anterior chamber of the eye [51], and this phenomenon was abrogated in the absence of B cells [52]. Studies suggested that splenic $B$ cells presented antigens derived from ocular APCs and induced $\mathrm{CD} 4{ }^{+} \mathrm{CD} 25^{+}$regulatory $\mathrm{T}$ cells via $\mathrm{IL}-10$ and $\mathrm{MHC}$ class II $[52,53]$. These evidence emphasize the role of $\mathrm{B}$ cells in the induction and maintenance of self-tolerance.

There is accumulating evidence demonstrating that specific B cell subsets modulate immune responses named as regulatory B (Breg) cells by Mizoguchi et al. [54]. Breg cells dampened immune responses though the secretion of IL-10, TGF- $\beta$, directly interact with activated $\mathrm{CD} 4^{+} \mathrm{T}$ cells, and the production of antibody that neutralized harmful soluble molecules [55]. Several Breg cells have been described in mice and IL-10-producing Breg cells are the most widely studied [56]. IL-10 produced by a variety of Breg cells suppressed inflammatory cytokines and promoted regulatory $\mathrm{T}$ cell differentiation $[57,58]$. These indicate that $\mathrm{B}$ cells contribute to the maintenance of tolerance.

In addition, naïve $\mathrm{B}$ cells functioned as antigenpresenting cells presented antigen and resulted in $\mathrm{T}$ cell tolerance to antigen [59]. Raimondi et al. demonstrated that adoptive transfer of antigen-presenting B cells four times in a week lead to antigen-specific $\mathrm{CD} 4^{+} \mathrm{T}$ cells tolerance independent of naïve or activated $B$ cells $[60,61]$. Antigen-presenting follicular B, marginal zone B, and B-1a cells rendered antigen-specific $\mathrm{T}$ cells hyporesponsiveness without Foxp3 ${ }^{+}$Treg cells induction [62]. One study reported that B cells contributed to Treg cells homeostasis and cooperated with Treg cells to ameliorate inflammation [63]. These findings suggest that B cells play a role in immune modulation and might through the manipulation of $\mathrm{CD}^{+}$Treg cells.

\section{B-cell-induced $\mathrm{CD}^{+}{ }^{+}$Foxp3 ${ }^{-}$Treg-of-B cells}

Naïve splenic B2 cells, peritoneal B-1a cells, and mucosal Peyer's patch $B$ cells have been shown to induce CD4 ${ }^{+} \mathrm{CD} 25^{+}$Foxp3 ${ }^{-}$regulatory $\mathrm{T}$ cells, which named Treg-of$\mathrm{B}$ cells by our group, without additional cytokines or molecules [50, 64]. Naïve splenic B cells and naïve splenic $\mathrm{CD} 4^{+} \mathrm{CD} 25^{-} \mathrm{T}$ cells formed a stable immunological synapse and promoted $\mathrm{CD} 62 \mathrm{~L}^{\text {hi }} \mathrm{CD} 25^{+}$Foxp $3^{-}$ regulatory $\mathrm{T}$ cell generation [65]. In our reports, transwell insertion during B-T coculture abrogated Treg-of-B cell induction suggesting that cell-cell contact between $\mathrm{B}$ and $\mathrm{T}$ cells was essential. By applying blocking antibodies during B-T coculture, both CD80 and CD86 on splenic $B$ cells were required to induce functional Treg-of-B [64]. In consistent with above, Etemire et al. demonstrated that addition of anti-CD28 antibody to the $\mathrm{B}-\mathrm{T}$ cell co-culture decreased the suppressive activity of Treg-of-B cells. Lower activity of the PI3K/AKT pathway was associated with Foxp3 $3^{-}$regulatory $\mathrm{T}$ cell generation [66]. IL-10-deficient Treg-of-B cells and Treg-of-B cells induced in the presence of anti-IL-10 neutralizing antibody remained their suppressive function suggesting that IL-10 was not critical for their induction $[64,67,68]$. These results suggest that the interaction between $\mathrm{B}-\mathrm{T}$ cells is indispensable for the differentiation of Treg-of-B cells.

\section{Treg-of-B cells differ from well-known Treg cells}

To date, several molecules have been identified for their strong association with Treg-of-B cells that are conserved in single peptide-induced and anti-CD3/CD28 antibodies-induced methods. Treg-of-B cells expressed higher levels of LAG3, ICOS, PD1, GITR, OX40 (CD134), and CTLA4 compared to those on naïve CD4 
${ }^{+} \mathrm{CD} 25^{-} \mathrm{T}$ cells (Fig. 1). Another group demonstrated that antigen-presenting $\mathrm{B}$ cells facilitated naïve $\mathrm{T}$ cells to convert into $\mathrm{CD}^{+} \mathrm{CD} 25^{+} \mathrm{CD} 2 \mathrm{~L}^{+} \mathrm{Foxp} 3^{-}$IL-10-producing regulatory $\mathrm{T}$ cells [65]. Our published and unpublished data showed that Treg-of-B cells did not express Foxp3, Helios, or neuropilin-1 $[67,69]$, and these also confirmed by using Foxp3-GFP reporter mice [64]. These evidence differentiates Treg-of-B cells from Foxp3-expressing Treg cells (Table 1).

Th3 cells are well-known that they exert TGF- $\beta$ dependent inhibition and express LAP on surface [26]. Although Treg-of-B cells produced TGF- $\beta$ compared with naïve $\mathrm{CD} 4^{+} \mathrm{CD} 25^{-} \mathrm{T}$ cells $[68,69]$, TGF- $\beta$ did not play a role in their suppressive mechanism [64]. In our unpublished data, Treg-of-B cells did not express LAP. These indicate that Treg-of-B cells are different from Th3 cells.

$\operatorname{Tr} 1$ cells are characterized by IL-10-mediated suppression and the higher production of IL-10 [27]. In recent years, CD49b, LAG3, and CD226 were identified as the surface markers for human and mouse Tr1 cells [35]. In our results, Treg-of- $\mathrm{B}$ cells produced a higher amount of IL-10 compared with naïve $\mathrm{CD} 4^{+} \mathrm{CD} 25^{-} \mathrm{T}$ cells $[50,64]$. Repeated stimulation of $\mathrm{B}$ cells induced long-term Tregof-B cells with higher expression of ICOS, CTLA4, $\mathrm{CD} 49 \mathrm{~b}$, and c-Maf, but not CD226. In addition to the difference in surface marker, IL-10 seems to be dispensable in the inhibitory mechanism of Treg-of-B cells and these would be described in the later section. These observations suggest that this Treg-of- $B$ cell is a new type of regulatory $\mathrm{T}$ cells and different from $\mathrm{Tr} 1$ cells.

In addition to regulatory $\mathrm{T}$ cells, Treg-of- $\mathrm{B}$ cells did not share characteristics with follicular $\mathrm{T}$ helper $\left(\mathrm{T}_{\mathrm{FH}}\right)$ cells. $\mathrm{T}_{\mathrm{FH}}$ cells expressed BCL-6, CXCR5, ICOS, PD1, and c-Maf and CXCR5 conferred $\mathrm{T}_{\mathrm{FH}}$ cells migration to B follicles [67, 70]. Although Treg-of-B cells expressed ICOS, PD1 and c-Maf, they did not express the critical molecule BCL-6 and CXCR5 (data not shown). These indicate that Treg-of-B cells could not migrate into follicle to facilitate $\mathrm{B}$ cell as $\mathrm{T}_{\mathrm{FH}}$ cells did.

Furthermore, Treg-of-B cells were hypoproliferative to stimulation and did not express T-bet, GATA3, or ROR$\gamma t$ ([64] and our unpublished data). Treg-of-B cells produced higher level of IL-10, TGF- $\beta$, and IL- 4 and lower or no IL-2, IFN- $\gamma$, IL-17, or tumor necrosis factor (TNF)- $\alpha[68,69,71]$. These data confirm that Treg-of-B cells have anergic characteristics and are not proinflammatory $\mathrm{T}$ helper cells.

\section{Application of Treg-of-B cells}

The therapeutic effects of $\mathrm{CD}^{+}{ }^{+}$Foxp3- Treg-of-B cells has been described in several murine disease models (Fig. 2). Adoptive transfer of Treg-of-B cells prevented mice from graft-versus-host disease in a murine model of heart transplantation [65]. Peyer's patch B-cellinduced ovalbumin (OVA)-specific Treg-of-B cells

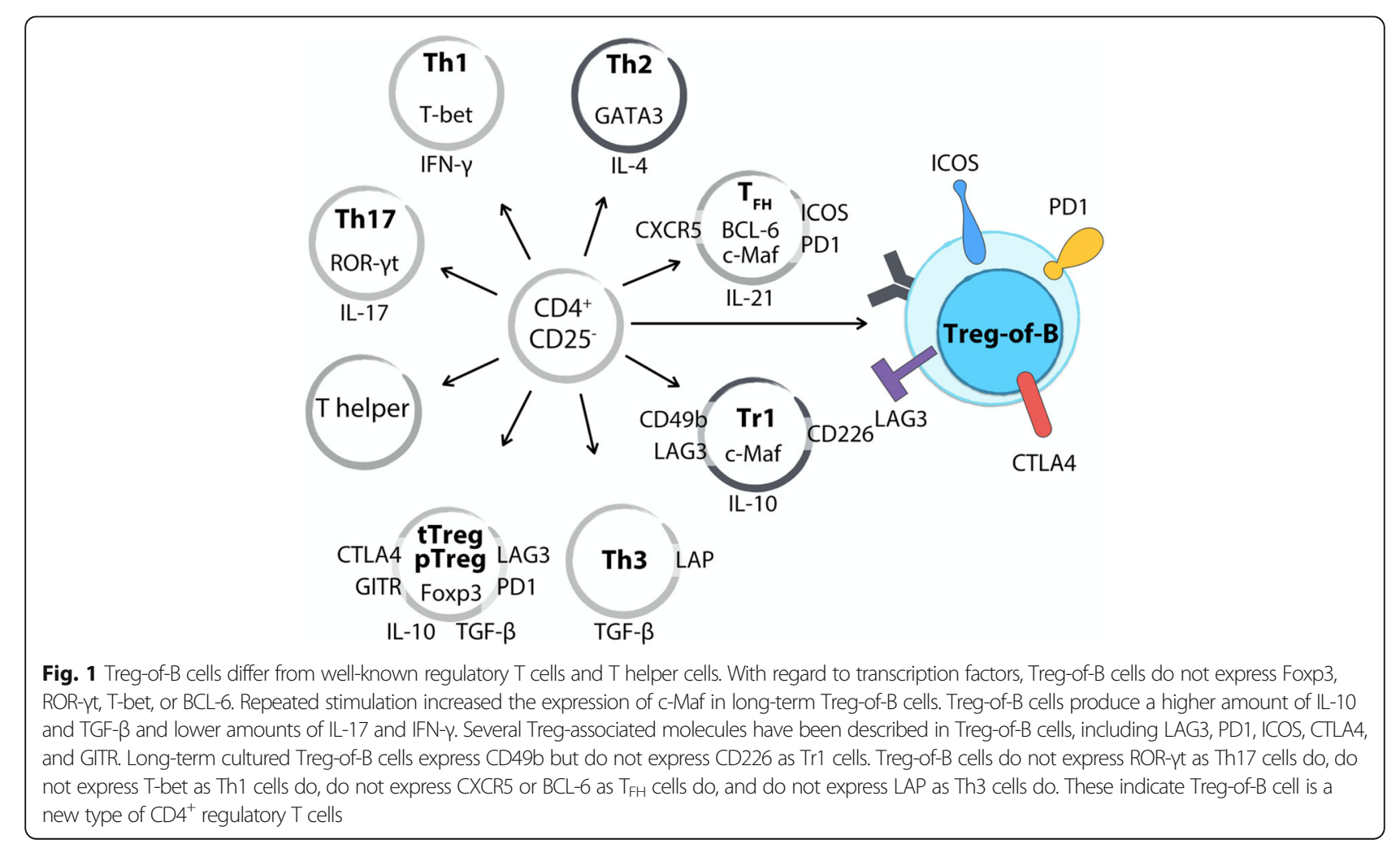


Table 1 The differences between Treg-of-B cells and the well-known Treg cells, including Foxp3 ${ }^{+} \operatorname{Treg}$, Th3, and Tr1 cells

\begin{tabular}{|c|c|c|c|c|}
\hline Treg cells & Biomarkers & Effector molecules & Transcription factors & Assisted cell types \\
\hline Treg-of-B & $\begin{array}{l}\mathrm{CD}^{+} \mathrm{CD} 25^{+} \mathrm{Foxp}^{-} \mathrm{LAG3}^{+} \text {ICOS } \\
{ }^{+} \mathrm{PD} 1^{+} \mathrm{GITR}^{+} \mathrm{OX}_{40}^{+}\end{array}$ & $\begin{array}{l}\text { Majorly contact-dependent } \\
\text { IL-10, LAG3, and CTLA4 has reported in reference }\end{array}$ & Undefined & B cells \\
\hline Foxp3 ${ }^{+}$Treg & $\begin{array}{l}\mathrm{CD}^{+} \text {Foxp } 3^{+} \\
\text {Helios has reported in reference }\end{array}$ & $\begin{array}{l}\text { IL-10, TGF- } \beta \text {, IL-35, LAP, CD39/CD73, CAMP, CTLA4, } \\
\text { LAG3, IL-2/IL-7 consumption, granzyme B, galectin- } \\
\text { 1, DR5...etC }\end{array}$ & Foxp3 & $\begin{array}{l}\text { DCs, macrophages, } \\
\text { B cells }\end{array}$ \\
\hline Th3 & $\mathrm{CD}^{+}{ }^{+}$Foxp3 ${ }^{-} \mathrm{LAP}^{+}$ & $\begin{array}{l}\text { Majorly TGF- } \beta \\
\text { IL-10 has reported in reference }\end{array}$ & Undefined & DCs \\
\hline $\operatorname{Tr} 1$ & $\begin{array}{l}\mathrm{CD}^{+}{ }^{+} \mathrm{Foxp}^{-} \mathrm{CD} 49 \mathrm{~b}^{+} \\
\mathrm{LAG}^{+} \mathrm{CD}^{+} 26^{+}\end{array}$ & $\begin{array}{l}\text { Majorly IL-10 } \\
\text { TGF- } \beta, \text { CTLA4, and CD226 has reported in } \\
\text { reference }\end{array}$ & $\begin{array}{l}\text { Undefined } \\
\text { c-Maf, AhR has reported } \\
\text { in reference }\end{array}$ & $\begin{array}{l}\text { DCs, macrophages, } \\
\text { B cells, mast cells...etc }\end{array}$ \\
\hline
\end{tabular}

protected mice from Th2-cell-mediated airway hyperresponsiveness (AHR), airway inflammation, and IgE hyperproduction in allergic asthma in an antigen-specific fashion [50]. In addition, splenic B-cell-induced OVA-specific Tregof-B cells shared several characteristics with oral antigen administration activated $\mathrm{CD} 4{ }^{+} \mathrm{CD} 25^{+} \mathrm{T}$ cells, including elevated expression levels of ICOS, PD1, and CTLA4 and enhanced non-antigen-specific suppressive functions [69]. Monoclonal antibody-induced Treg-of-B cells prevented mice from osteolysis and joint inflammation in collageninduced arthritis [71]. Prophylactic transfer of Treg-of-B cells also protected mice from T-cell-induced Th1- and Th17-dominant inflammatory bowel disease [68]. Taken together, naïve B cell without cytokines or chemical supplements is able to induce functional $\mathrm{CD} 4^{+} \mathrm{Foxp}^{-}$regulatory $\mathrm{T}$ cells and that B-cell-induced regulatory $\mathrm{T}$ cells is an economical strategy for cellular therapy for different T-helpercell-dominant inflammatory diseases.
Treg-of-B cells possess both IL-10-dependent and IL-10independent suppressive functions

IL-10 as an anti-inflammatory cytokine is an issue in Treg-of-B cells suppressive function. As described above, IL-10 does not play a crucial role in Treg-of-B cells differentiation. Chen and $\mathrm{Chu}$ et al. reported that LAG3 ${ }^{+}$Treg-of-B cells produced higher amount of IL-10 and both IL-10 and LAG3 play the roles in their inhibitory mechanisms [71, 72]. Long-term Treg-of-B cells increased expression levels of CTLA4 and IL-10, both of which were involved in their suppressive functions [67]. IL-10-deficient mice were used to confirm the role of IL-10 in the regulation; however, IL-10-deficient Tregof-B cells remained suppressive activities [64, 68]. IL-10 seems to be dispensable in the inhibitory mechanism of Treg-of-B cells. Although IL-10 plays a more important role in long-term Treg-of-B cells than in short-term Treg-of-B cells, three-day short-term culture is sufficient

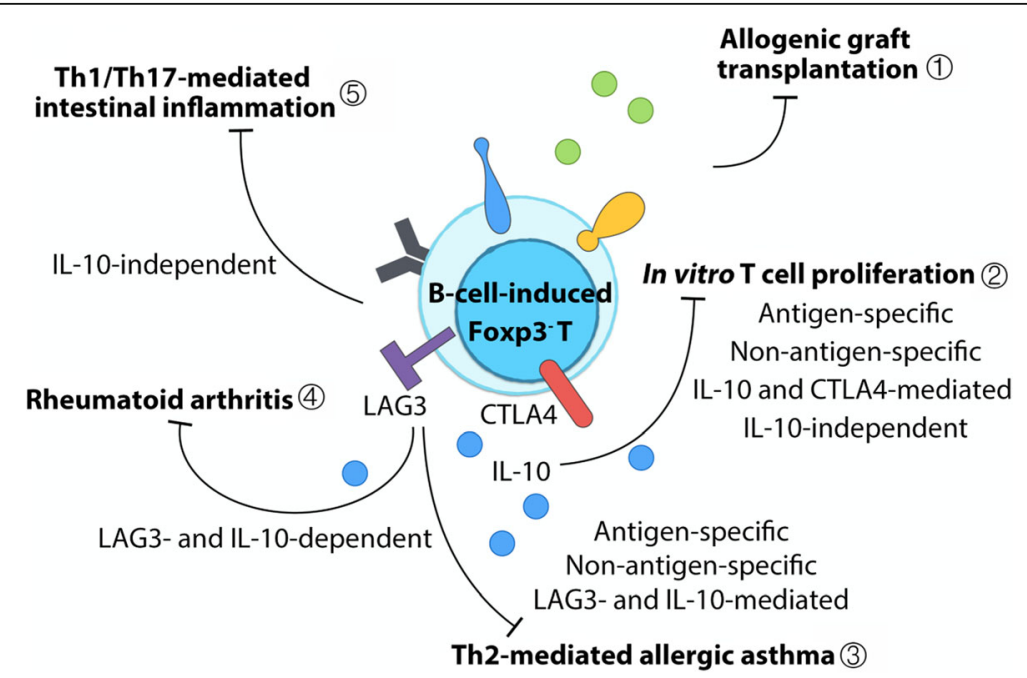

Fig. 2 B-cell-induced CD4 ${ }^{+}$Foxp3 ${ }^{-}$regulatory T cells treatment in disease models. Treg-of-B cells have been used for therapy in several animal models. Pre-treated Treg-of-B cells prevented allogeneic heart transplantation-induced tissue rejection (1). Treg-of-B cells inhibited antigen-specific and non-antigen-specific T cell proliferation in vitro through IL-10-mediated and IL-10-independent mechanisms. Both IL-10 and CTLA4 play roles in long-term Treg-of-B cells suppressive functions (2). In vivo treatment with Treg-of-B cells protected mice from Th2-mediated allergic asthma in an antigen-specific manner and in a non-antigen-specific fashion (3). Both LAG3 and IL-10 may play roles in the protection of mice from allergic asthma and rheumatoid arthritis (4). IL-10-deficient Treg-of-B cells prevented mice from T-cell-mediated intestinal inflammation (5) 
for the generation of Treg-of-B cells. These suggest that there might be unknown inhibitory factors in Treg-of-B cells suppressive functions.

Studies have demonstrated that ICOS controls IL-10 production and functional CTLA4 expression in Treg cells [73-75]. PD1 recruits SHP-1 and SHP-2 to intrinsically downregulate $\mathrm{T}$ cell receptor signaling, which maintains an anergic phenotype in Treg cells [76, 77]. Mouse Treg cells constitutively expressed GITR and OX40 and involved the tTreg cells development as well as their functions [78-80]. All regulatory-T-related molecules on Treg-of-B cells, including IL-10, TGF- $\beta$, LAG3, CTLA4, ICOS, PD1, GITR, and OX40, might confer partial suppressive activities to compensate for single blockage or neutralization. The critical molecules controlling Treg-of-B cell phenotype and regulatory mechanisms remain priorities for investigation. The inhibitory functions of Treg-of-B cell depend on the suppressive molecules on the surface or soluble mediators that require short distance.

\section{B-cell-induced $\mathrm{CD}^{+}{ }^{+} \mathrm{Foxp}^{+}{ }^{+}$regulatory $\mathrm{T}$ cells}

Reports have revealed the role of $\mathrm{B}$ cells in the development of Treg cells. Naïve primary B cells preferentially induced the expansion of allogenic $\mathrm{CD} 4^{+} \mathrm{Foxp}^{+} \mathrm{T}$ cells rather than $\mathrm{CD}^{+}{ }^{+}$Foxp3 ${ }^{-} \mathrm{T}$ cells $[81,82]$. Splenic B cells converted allogenic naïve $\mathrm{T}$ cells into Foxp $3^{+}$regulatory $\mathrm{T}$ cells in the presence of TGF- $\beta$ and IL-2, and peritoneal B cells induce Th17 cells [83]. Human CD40activated $\mathrm{B}$ cells induced the differentiation of CD25 ${ }^{+}$Foxp $3{ }^{+} \mathrm{CD} 62 \mathrm{~L}^{+}$regulatory $\mathrm{T}$ cells more efficiently than immature DCs $[84,85]$. In contrast, reports demonstrated that murine CD40-activated B cells promoted $\mathrm{CD}^{+} \mathrm{T}$ cell proliferation and effector functions [86, 87]. Furthermore, the frequency of intrathymic B cells correlated with that of tTreg cells, and B cells colocalized with tTreg cells in the thymus $[88,89]$. Intrathymic B cells expressed autoimmune regulator (Aire), increased the levels of MHC class II and CD80, and contributed to $\mathrm{T}$ cell negative selection for central $\mathrm{T}$ cell tolerance [90, 91]. Taken together, there are unknown criteria, such as MHC class II-TCR signaling, the B cell activation status, and different types of tissue resident $\mathrm{B}$ cells, that may fine-tune the expression of Foxp3 in B-cellinduced regulatory $\mathrm{T}$ cells.

\section{Conclusions}

To date, we know that naive antigen-presenting B cell is sufficient to induce $\mathrm{CD} 4^{+}{ }^{-}$oxp $3^{-}$regulatory $\mathrm{T}$ cells without additional cytokines or chemicals in an IL-10- and IL-27-dispensable and cell-cell contact-dependent manner. The expression levels of characteristic molecules differentiate Treg-of- $B$ cells from well-known $T$ helper and regulatory $\mathrm{T}$ cells as a brand-new type of $\mathrm{CD} 4$
${ }^{+}$Foxp3 ${ }^{-}$regulatory $\mathrm{T}$ cells (Fig. 1). Treg-of-B cells possess IL-10-depedent, IL-10-independent, and cell-cell contact-dependent suppressive abilities in antigenspecific and non-antigen specific fashions. Compared to long-term Treg-of-B cells, short-term Treg-of-B cells act through multiple suppressive pathways, and thus a blockade strategy would be more easily overcome through compensation by other pathways. Treg-of-B cells exhibit immunomodulatory effects in Th2-, Th1-, and Th17-medated diseases and even allogeneic transplantation. Nevertheless, the physiological conditions or cues necessary for Treg-of-B cell generation remain unknown. What is the fine-tuning mechanism for B cells to induce $\mathrm{CD}^{+} \mathrm{Foxp}^{-}$or expand $\mathrm{CD} 4^{+} \mathrm{Foxp}^{+}{ }^{+} \mathrm{T}$ cells? What factors determine the kinetics, memory, and maintenance? And, most importantly, how could we use Treg-of-B cells in immunotherapy?

\section{Abbreviations \\ Breg: Regulatory B; Foxp3: Forkhead box P3; ICOS: Inducible T-cell co-stimulator; IL-10: Interleukin 10; iTreg: in vitro-induced Treg; LAG3: Lymphocyte-activation gene 3; PD1: Programmed cell death protein 1; pTreg: Peripherally derived regulatory T; TGF- $\beta$ : Transforming growth factor- $\beta$; Th3: Type 3 helper; Tr1: Type 1 regulatory T; Treg: Regulatory T; Treg-of-B: B cell-induced regulatory T; tTreg: Thymus-derived regulatory $T$ \\ Acknowledgements \\ None. \\ Funding \\ This study was supported by the grant from Excellent Translational Medicine Research Projects of National Taiwan University College of Medicine and National Taiwan University Hospital (104C101-81).}

Availability of data and materials

Not applicable.

\section{Authors' contributions}

C-H C performed the literature reviewed and drafted the manuscript. B-L C supervised and critically reviewed the manuscript. Both authors read and approved the final manuscript.

\section{Authors' information \\ None.}

Ethics approval and consent to participate Not applicable.

\section{Consent for publication \\ None.}

\section{Competing interests}

The authors declare that they have no competing interests.

\section{Publisher's Note}

Springer Nature remains neutral with regard to jurisdictional claims in published maps and institutional affiliations.

Received: 7 July 2017 Accepted: 3 November 2017

Published online: 18 November 2017

\section{Reference}

1. Sakaguchi S, et al. Immunologic self-tolerance maintained by activated T cells expressing IL-2 receptor alpha-chains (CD25). Breakdown of a 
single mechanism of self-tolerance causes various autoimmune diseases. J Immunol. 1995;155(3):1151-64.

2. Wildin RS, et al. X-linked neonatal diabetes mellitus, enteropathy and endocrinopathy syndrome is the human equivalent of mouse scurfy. Nat Genet. 2001;27(1):18-20.

3. Bennett $\mathrm{CL}$, et al. The immune dysregulation, polyendocrinopathy, enteropathy, $\mathrm{X}$-linked syndrome (IPEX) is caused by mutations of FOXP3. Nat Genet. 2001;27(1):20-1.

4. Tommasini $\mathrm{A}$, et al. X-chromosome inactivation analysis in a female carrier of FOXP3 mutation. Clin Exp Immunol. 2002;130(1):127-30.

5. Hori S, Nomura T, Sakaguchi S. Control of regulatory T cell development by the transcription factor Foxp3. Science. 2003;299(5609):1057-61.

6. Sugimoto N, et al. Foxp3-dependent and -independent molecules specific for CD25+CD4+ natural regulatory T cells revealed by DNA microarray analysis. Int Immunol. 2006;18(8):1197-209.

7. Thornton AM, Shevach EM. Suppressor effector function of CD4+CD25+ immunoregulatory T cells is antigen nonspecific. J Immunol. 2000; 164(1):183-90

8. Collison LW, et al. The inhibitory cytokine IL-35 contributes to regulatory T-cell function. Nature. 2007;450(7169):566-9.

9. Nakamura K, Kitani A, Strober W. Cell contact-dependent immunosuppression by CD4(+)CD25(+) regulatory $T$ cells is mediated by cell surface-bound transforming growth factor Beta. J Exp Med. 2001;194(5):629-44

10. Deaglio $\mathrm{S}$, et al. Adenosine generation catalyzed by CD39 and CD73 expressed on regulatory $T$ cells mediates immune suppression. J Exp Med. 2007;204(6):1257-65.

11. Bopp T, et al. Cyclic adenosine monophosphate is a key component of regulatory T cell-mediated suppression. J Exp Med. 2007;204(6):1303-10.

12. Pandiyan $P$, et al. CD4+CD25+Foxp3+ regulatory $T$ cells induce cytokine deprivation-mediated apoptosis of effector CD4+ T cells. Nat Immunol. 2007;8(12):1353-62.

13. Gondek DC, et al. Cutting edge: contact-mediated suppression by CD4 +CD25+ regulatory cells involves a granzyme B-dependent, perforinindependent mechanism. J Immunol. 2005;174(4):1783-6.

14. Garin Ml, et al. Galectin-1: a key effector of regulation mediated by CD4 +CD25+ T cells. Blood. 2007;109(5):2058-65.

15. Ren $\mathrm{X}$, et al. Involvement of cellular death in TRAIL/DR5-dependent suppression induced by CD4(+)CD25(+) regulatory T cells. Cell Death Differ. 2007;14(12):2076-84

16. Wing $\mathrm{K}$, et al. CTLA-4 control over Foxp3+ regulatory $T$ cell function. Science. 2008;322(5899):271-5.

17. Liang $B$, et al. Regulatory $T$ cells inhibit dendritic cells by lymphocyte activation gene-3 engagement of MHC class II. J Immunol. 2008; 180(9):5916-26.

18. Abbas AK, et al. Regulatory $T$ cells: recommendations to simplify the nomenclature. Nat Immunol. 2013;14(4):307-8.

19. Chen W, et al. Conversion of peripheral CD4+CD25- naive T cells to CD4 +CD25+ regulatory $T$ cells by TGF-beta induction of transcription factor Foxp3. J Exp Med. 2003;198(12):1875-86.

20. Huter EN, et al. TGF-beta-induced Foxp3+ regulatory T cells rescue scurfy mice. Eur J Immunol. 2008;38(7):1814-21.

21. Mucida D, et al. Oral tolerance in the absence of naturally occurring Tregs. J Clin Invest. 2005;115(7):1923-33.

22. Coombes $\mathrm{J}$, et al. A functionally specialized population of mucosal CD103+ DCs induces Foxp3+ regulatory $T$ cells via a TGF-beta and retinoic aciddependent mechanism. J Exp Med. 2007;204(8):1757-64.

23. Soroosh $P$, et al. Lung-resident tissue macrophages generate Foxp3+ regulatory $T$ cells and promote airway tolerance. J Exp Med. 2013; 210(4):775-88.

24. Cao X, et al. Granzyme B and perforin are important for regulatory T cellmediated suppression of tumor clearance. Immunity. 2007;27(4):635-46.

25. Yadav $M$, et al. Neuropilin-1 distinguishes natural and inducible regulatory $T$ cells among regulatory $T$ cell subsets in vivo. J Exp Med. 2012;209(10):1713-22. S1-19

26. Weiner HL, et al. Oral tolerance. Immunol Rev. 2011;241(1):241-59.

27. Zeng $\mathrm{H}$, et al. Type 1 regulatory $T$ cells: a new mechanism of peripheral immune tolerance. Cell Mol Immunol. 2015;12(5):566-71.

28. Chen $Y$, et al. Regulatory $T$ cell clones induced by oral tolerance: suppression of autoimmune encephalomyelitis. Science. 1994; 265(5176):1237-40
29. Oida T, et al. CD4+CD25- T cells that express latency-associated peptide on the surface suppress CD4+CD45RBhigh-induced colitis by a TGF-beta-dependent mechanism. J Immunol. 2003;170(5):2516-22.

30. Han Y, et al. CD69+ CD4+ CD25-T cells, a new subset of regulatory T cells, suppress T cell proliferation through membrane-bound TGF-beta 1. J Immunol. 2009;182(1):111-20.

31. Gandhi R, et al. Cutting edge: human latency-associated peptide+ T cells: a novel regulatory T cell subset. J Immunol. 2010;184(9):4620-4.

32. Scurr $M$, et al. Highly prevalent colorectal cancer-infiltrating LAP(+) Foxp3(-) T cells exhibit more potent immunosuppressive activity than Foxp3(+) regulatory T cells. Mucosal Immunol. 2014;7(2):428-39.

33. Groux H, et al. A CD4+ T-cell subset inhibits antigen-specific T-cell responses and prevents colitis. Nature. 1997;389(6652):737-42.

34. Akbari $\mathrm{O}$, et al. Antigen-specific regulatory $T$ cells develop via the ICOSICOS-ligand pathway and inhibit allergen-induced airway hyperreactivity. Nat Med. 2002;8(9):1024-32.

35. Gagliani N, et al. Coexpression of CD49b and LAG-3 identifies human and mouse T regulatory type 1 cells. Nat Med. 2013;19(6):739-46.

36. $\mathrm{Xu} \mathrm{J}$, et al. C-Maf regulates IL-10 expression during Th17 polarization. J Immunol. 2009;182(10):6226-36.

37. Apetoh $L$, et al. The aryl hydrocarbon receptor interacts with c-Maf to promote the differentiation of type 1 regulatory $T$ cells induced by IL-27. Nat Immunol. 2010;11(9):854-61.

38. Awasthi $A$, et al. A dominant function for interleukin 27 in generating interleukin 10-producing anti-inflammatory T cells. Nat Immunol. 2007; 8(12):1380-9.

39. Pot C, et al. Cutting edge: IL-27 induces the transcription factor C-Maf, cytokine IL-21, and the costimulatory receptor ICOS that coordinately act together to promote differentiation of IL-10-producing Tr1 cells. J Immunol. 2009:183(2):797-801.

40. Wu HY, et al. In vivo induction of $\operatorname{Tr} 1$ cells via mucosal dendritic cells and AHR signaling. PLoS One. 2011;6(8):e23618.

41. Shiokawa A, et al. IL-10 and IL-27 producing dendritic cells capable of enhancing IL-10 production of T cells are induced in oral tolerance. Immunol Lett. 2009;125(1):7-14.

42. Carrier $Y$, et al. Enhanced GITR/GITRL interactions augment IL-27 expression and induce IL-10-producing Tr-1 like cells. Eur J Immunol. 2012:42(6):1393-404.

43. Cedeno-Laurent $F$, et al. Galectin-1 triggers an immunoregulatory signature in Th cells functionally defined by IL-10 expression. J Immunol. 2012;188(7):3127-37.

44. Yao Y, et al. Tr1 Cells, but Not Foxp3+ Regulatory T Cells, Suppress NLRP3 Inflammasome Activation via an IL-10-Dependent Mechanism. J Immunol. 2015;195(2):488-97.

45. Nie $X$, et al. Lipopolysaccharide mediated mast cells induce IL-10 producing regulatory $T$ cells through the ICOSL/ICOS axis. Clin Immunol. 2012;142(3):269-79.

46. Wolf SD, et al. Experimental autoimmune encephalomyelitis induction in genetically B cell-deficient mice. J Exp Med. 1996;184(6):2271-8.

47. Andersson SE, et al. Collagen epitope expression on B cells is sufficient to confer tolerance to collagen-induced arthritis. Arthritis Res Ther. 2016;18(1):140.

48. Gonnella PA, Waldner HP, Weiner HL. B cell-deficient (mu MT) mice have alterations in the cytokine microenvironment of the gut-associated lymphoid tissue (GALT) and a defect in the low dose mechanism of oral tolerance. J Immunol. 2001;166(7):4456-64.

49. Gutgemann I, et al. A blood-borne antigen induces rapid T-B cell contact: a potential mechanism for tolerance induction. Immunology. 2002;107(4):420-5.

50. Chu KH, Chiang BL. Regulatory T cells induced by mucosal B cells alleviate allergic airway hypersensitivity. Am J Respir Cell Mol Biol. 2012; 46(5):651-9.

51. D'Orazio TJ, Niederkorn JY. Splenic B cells are required for tolerogenic antigen presentation in the induction of anterior chamber-associated immune deviation (ACAID). Immunology. 1998;95(1):47-55

52. Skelsey ME, Mayhew E, Niederkorn JY. Splenic B cells act as antigen presenting cells for the induction of anterior chamber-associated immune deviation. Invest Ophthalmol Vis Sci. 2003:44(12):5242-51.

53. Ashour HM, Niederkorn JY. Peripheral tolerance via the anterior chamber of the eye: role of B cells in MHC class I and II antigen presentation. J Immunol. 2006;176(10):5950-7. 
54. Mizoguchi A, et al. Chronic intestinal inflammatory condition generates IL-10-producing regulatory B cell subset characterized by CD1d upregulation. Immunity. 2002;16(2):219-30.

55. Mizoguchi A, Bhan AK. A case for regulatory B cells. J Immunol. 2006; 176(2):705-10.

56. Miyagaki T, Fujimoto M, Sato S. Regulatory B cells in human inflammatory and autoimmune diseases: from mouse models to clinical research. Int Immunol. 2015;27(10):495-504.

57. Mauri C, Bosma A. Immune regulatory function of B cells. Annu Rev Immunol. 2012;30:221-41.

58. Gray $\mathrm{M}$, et al. Apoptotic cells protect mice from autoimmune inflammation by the induction of regulatory B cells. Proc Natl Acad Sci U S A. 2007; 104(35):14080-5.

59. Eynon EE, Parker DC. Small B cells as antigen-presenting cells in the induction of tolerance to soluble protein antigens. J Exp Med. 1992; 175(1):131-8.

60. Raimondi $\mathrm{G}$, et al. Induction of peripheral T cell tolerance by antigenpresenting B cells. II. Chronic antigen presentation overrules antigenpresenting B cell activation. J Immunol. 2006;176(7):4021-8.

61. Raimondi $\mathrm{G}$, et al. Induction of peripheral T cell tolerance by antigenpresenting B cells. I. Relevance of antigen presentation persistence. J Immunol. 2006;176(7):4012-20.

62. Murray SE, Toren KG, Parker DC. Peripheral CD4(+) T-cell tolerance is induced in vivo by rare antigen-bearing B cells in follicular, marginal zone, and B-1 subsets. Eur J Immunol. 2013:43(7):1818-27.

63. Wang $L$, et al. T regulatory cells and $B$ cells cooperate to form a regulatory loop that maintains gut homeostasis and suppresses dextran sulfate sodium-induced colitis. Mucosal Immunol. 2015;8(6):1297-312.

64. Hsu LH, et al. A B-1a cell subset induces Foxp3(-) T cells with regulatory activity through an IL-10-independent pathway. Cell Mol Immunol. 2015; 12(3):354-65.

65. Reichardt $P$, et al. Naive $B$ cells generate regulatory $T$ cells in the presence of a mature immunologic synapse. Blood. 2007;110(5):1519-29.

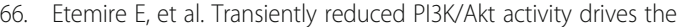
development of regulatory function in antigen-stimulated naive T-cells. PLoS One. 2013:8(7):e68378.

67. Chien $\mathrm{CH}$, et al. Characterization of c-Maf+Foxp3- regulatory T cells induced by repeated stimulation of antigen-presenting B cells. Sci Rep. 2017;7:46348,

68. Shao TY, et al. Novel Foxp3(-) IL-10(-) regulatory T-cells induced by B-cells alleviate intestinal inflammation in vivo. Sci Rep. 2016;6:32415.

69. Chien $\mathrm{CH}, \mathrm{Yu} \mathrm{HH}$, Chiang BL. Single allergen-induced oral tolerance inhibits airway inflammation in conjugated allergen immunized mice. J Allergy Clin Immunol. 2015;136(4):1110-3. e4

70. Qi H. T follicular helper cells in space-time. Nat Rev Immunol. 2016; 16(10):612-25

71. Chen SY, et al. Lymphocyte-activation gene 3(+) (LAG3(+)) forkhead box protein $3(-)$ (FOXP3 $(-))$ regulatory $T$ cells induced by $B$ cells alleviates joint inflammation in collagen-induced arthritis. J Autoimmun. 2016;68:75-85.

72. Chu KH, Chiang BL. Characterization and functional studies of forkhead box protein 3(-) lymphocyte activation gene 3(+) CD4(+) regulatory T cells induced by mucosal B cells. Clin Exp Immunol. 2015;180(2):316-28.

73. Kohyama M, et al. Inducible costimulator-dependent IL-10 production by regulatory T cells specific for self-antigen. Proc Natl Acad Sci U S A. 2004; 101(12):4192-7.

74. Tuettenberg $A$, et al. The role of ICOS in directing $T$ cell responses: ICOS-dependent induction of T cell anergy by tolerogenic dendritic cells. J Immunol. 2009;182(6):3349-56.

75. Zheng J, et al. ICOS regulates the generation and function of human CD4+ Treg in a CTLA-4 dependent manner. PLoS One. 2013;8(12):e82203.

76. Chemnitz JM, et al. SHP-1 and SHP-2 associate with immunoreceptor tyrosine-based switch motif of programmed death 1 upon primary human $T$ cell stimulation, but only receptor ligation prevents $T$ cell activation. J Immunol. 2004;173(2):945-54.

77. Fife BT, et al. Interactions between PD-1 and PD-L1 promote tolerance by blocking the TCR-induced stop signal. Nat Immunol. 2009;10(11):1185-92.

78. Ronchetti $S$, et al. Glucocorticoid-induced tumour necrosis factor receptor-related protein: a key marker of functional regulatory $T$ cells. J Immunol Res. 2015;2015:171520.

79. Griseri T, et al. OX40 is required for regulatory T cell-mediated control of colitis. J Exp Med. 2010;207(4):699-709.
80. Mahmud SA, et al. Costimulation via the tumor-necrosis factor receptor superfamily couples TCR signal strength to the thymic differentiation of regulatory T cells. Nat Immunol. 2014;15(5):473-81.

81. Chen $X$, Jensen PE. Cutting edge: primary B lymphocytes preferentially expand allogeneic FoxP3+ CD4 T cells. J Immunol. 2007;179(4):2046-50.

82. Chen LC, et al. Direct expansion of human allospecific FoxP3+CD4+ regulatory T cells with allogeneic B cells for therapeutic application. J Immunol. 2009;183(6):4094-102.

83. Zhong $X$, et al. Reciprocal generation of Th1/Th17 and T(reg) cells by B1 and B2 B cells. Eur J Immunol. 2007;37(9):2400-4.

84. Tu W, et al. Efficient generation of human alloantigen-specific CD4+ regulatory $T$ cells from naive precursors by CD40-activated B cells. Blood. 2008;112(6):2554-62.

85. Zheng J, et al. CD40-activated B cells are more potent than immature dendritic cells to induce and expand CD4(+) regulatory T cells. Cell Mol Immunol. 2010;7(1):44-50.

86. Liebig TM, et al. Murine model of CD40-activation of B cells. J Vis Exp. 2010; (37).

87. Wennhold K, et al. CD40-activated B cells induce anti-tumor immunity in vivo. Oncotarget. 2017:8(17):27740-53.

88. Ray A, et al. A novel IL-10-independent regulatory role for $B$ cells in suppressing autoimmunity by maintenance of regulatory T cells via GITR ligand. J Immunol. 2012;188(7):3188-98.

89. Walters $\mathrm{SN}$, et al. A role for intrathymic B cells in the generation of natural regulatory T cells. J Immunol. 2014;193(1):170-6.

90. Yamano $T$, et al. Thymic B cells are licensed to present self antigens for central T cell tolerance induction. Immunity. 2015;42(6):1048-61.

91. Lu FT, et al. Thymic B cells promote thymus-derived regulatory $T$ cell development and proliferation. J Autoimmun. 2015;61:62-72.

\section{Submit your next manuscript to BioMed Central and we will help you at every step:}

- We accept pre-submission inquiries

- Our selector tool helps you to find the most relevant journal

- We provide round the clock customer support

- Convenient online submission

- Thorough peer review

- Inclusion in PubMed and all major indexing services

- Maximum visibility for your research

Submit your manuscript at www.biomedcentral.com/submit 